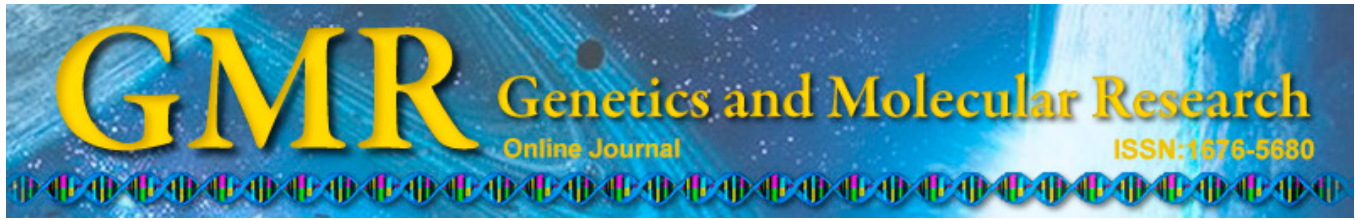

\title{
Highly efficient one-step PCR-based mutagenesis technique for large plasmids using high-fidelity DNA polymerase
}

\author{
H. Liu, R. Ye and Y.Y. Wang \\ Department of Medical Microbiology and Parasitology, \\ School of Basic Medical Sciences, Fudan University, Shanghai, China \\ Correspondence author: Y.Y. Wang \\ E-mail: yuyanss@fudan.edu.cn
}

Genet. Mol. Res. 14 (2): 3466-3473 (2015)

Received March 21, 2014

Accepted October 11, 2014

Published April 15, 2015

DOI http://dx.doi.org/10.4238/2015.April.15.10

\begin{abstract}
Gene mutation plays an important role in molecular biological studies. A highly efficient one-step polymerase chain reaction-based mutagenesis technique for site-directed mutagenesis was developed in this study. One complementary pair of primers was designed that contained the desired mutations in the middle of the primers. The amplification products of mutation were amplified using a high-fidelity DNA polymerase and the original plasmid templates were digested by $D p n$ I. This method was successfully used to introduce mutations in two different-sized plasmids (12 and $6 \mathrm{~kb})$ with high efficiency. The results indicate that this technique can be widely used to introduce any plasmid mutations quickly and efficiently.
\end{abstract}

Key words: $D p n$ I digestion; High-fidelity DNA polymerase; Site-directed mutagenesis 


\section{INTRODUCTION}

Site-directed mutagenesis is the foundation of modern molecular biology and genetic engineering and is widely used to study protein structure-function relationships. Directed mutagenesis includes adding and deleting bases as well as point mutations by changing a particular nucleotide sequence to precisely alter the protein structure. Currently, site-directed mutagenesis involves non-polymerase chain reaction (PCR)- and PCR-based techniques (Gillam et al., 1975; Zoller and Smith, 1987; Qi and Scholthof, 2008; Wu et al., 2013). PCR-mediated mutagenesis techniques are simple, fast, and efficient, and the Taq DNA polymerase that is typically used in PCR can result in mismatch, affecting the accuracy of mutation (Shinde et al., 2003).

Currently, the most widely used strategy for introducing mutations is the QuickChange $^{\mathrm{TM}}$ Site-Directed Mutagenesis Kit (Stratagene, La Jolla, CA, USA). Using this method, the vector containing the mutated bases is amplified by one pair of completely complementary primers, and then the methylated DNA template is extracted from Escherichia coli and digested by the restriction enzyme $D p n$ I. The PCR products that do not contain methylation sites cannot be digested by $D p n I$, and thus the products can be directly transformed into $E$. coli to obtain the desired vector containing the point mutation. High-fidelity Taq polymerase is typically used in this procedure. Though kits are convenient and involve simple operation steps, they are also expensive. In this study, some of the reagents and reaction conditions were modified and optimized to establish a simple, rapid, and economical method of site-directed mutagenesis.

In our protocol, a high-fidelity DNA polymerase to PCR-amplify the mutated product was used, and other reagents included routine laboratory reagents. Q5 ${ }^{\mathrm{TM}}$ High-fidelity DNA polymerase (New England Biolabs, Ipswich, MA, USA) is a high-fidelity, thermostable DNA polymerase with $3^{\prime} \rightarrow 5^{\prime}$ exonuclease activity, fused to a processivity-enhancing Sso7d domain to support robust DNA amplification. Because of its low error rate, Q5 High-fidelity DNA polymerase is ideal for cloning and can be used for long or difficult amplicons. Using $\mathrm{Q}^{\mathrm{TM}}$ High-fidelity DNA polymerase, we successfully amplified gene sequences up to $12 \mathrm{~kb}$ using site-directed mutagenesis. In addition, we compared the method with the Taq DNA polymerase from another company to determine error rates, costs, and DNA product quality. Our data suggest that the Q5 ${ }^{\mathrm{TM}}$ High-fidelity DNA polymerase method we developed is an excellent alternative for site-directed mutagenesis for long DNA fragments up to $12 \mathrm{~kb}$.

\section{MATERIAL AND METHODS}

\section{Preparation of template plasmid}

The pMH54-cmS, a 12-kb plasmid inserted by $\mathrm{S}$ gene fragments of canine coroanvirus, pIR-tN-EGFP $(6 \mathrm{~kb})$ inserted by $\mathrm{N}$ gene fragments of transmissible gastroenteritis virus, were used as the mutagenesis templates. The 2 plasmids were propagated in DH5 $\alpha$ E. coli cells (Tiangen Biotech, Beijing, China), and prepared using Qiagen miniprep kits (Qiagen, Hilden, Germany).

\section{Primer design}

The 3 pairs of mutagenic oligonucleotide primers used in this study are listed in Table 1 , which were designed to amplify the mutant fragments. These primer pairs contained com- 
pletely complementary sequences, with mutated bases at the center position. Three different positions from the two different plasmids were selected for mutagenesis by primer design. The primer pairs used in this experiment were completely complementary. Primers $\mathrm{tN}-501 \mathrm{~F}$, $\mathrm{tN}-501 \mathrm{R}, \mathrm{tN}-959 \mathrm{~F}$, and $\mathrm{tN}-959 \mathrm{R}$ were used to amplify the corresponding fragment for mutant pIR-tN ${ }^{501}$-EGFP and pIR-tN ${ }^{959}$-EGFP, while MH-1700F and MH-1700R were designed for pMH54-cmS ${ }^{1700}$ (Figure 1). All primers were synthesized and purified by Sangon Biotech (Shanghai, China). The NEB Tm Calculator was used to determine the optimal annealing temperatures for the primers.

Table 1. Primer sequences used in this study.

\begin{tabular}{ll}
\hline Primer name & Sequence $\left(5^{\prime} \rightarrow 3^{\prime}\right)$ \\
\hline $\mathrm{tN}-501 \mathrm{~F}$ & ACGCTCTCAATCTAGATCTCGGTCTAGAAAT \\
$\mathrm{tN}-501 \mathrm{R}$ & ATTTCTAGACCGAGATCTAGATTGAGAGCGT \\
$\mathrm{tN}-959 \mathrm{~F}$ & ATGATCCTAAGACTGAACAATTCCTTCAGCA \\
$\mathrm{tN}-959 \mathrm{R}$ & TGCTGAAGGAATTGTTCAGTCTTAGGATCAT \\
$\mathrm{MH}-1700 \mathrm{~F}$ & TAGCTGTGTTGATTATTGTGTTTTTGATG \\
$\mathrm{MH}-1700 \mathrm{R}$ & CATCAAAAACACAATAATCAACACAGCTA \\
\hline
\end{tabular}

Underlined letters are the mutated bases.

A
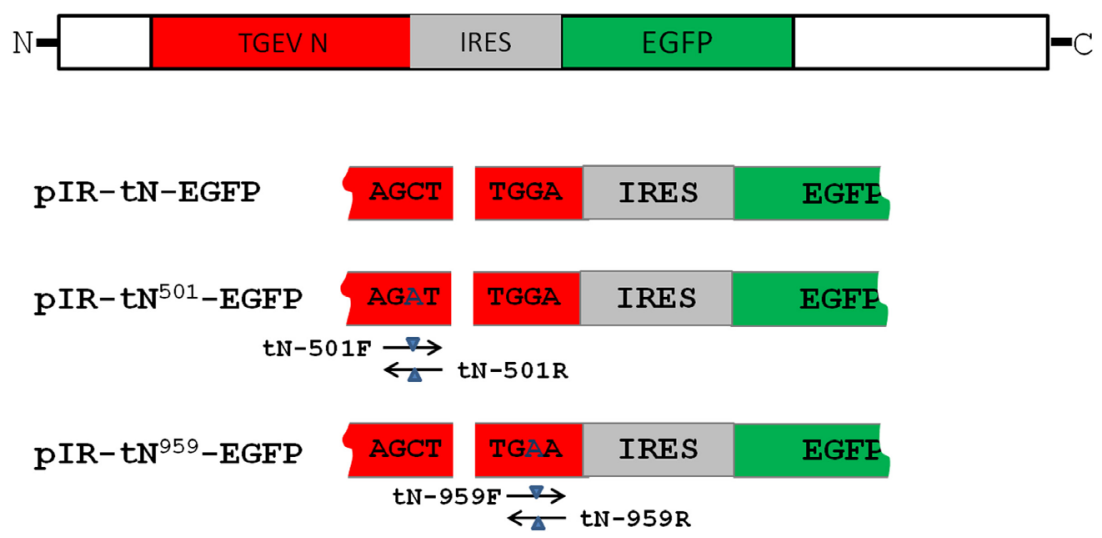

B

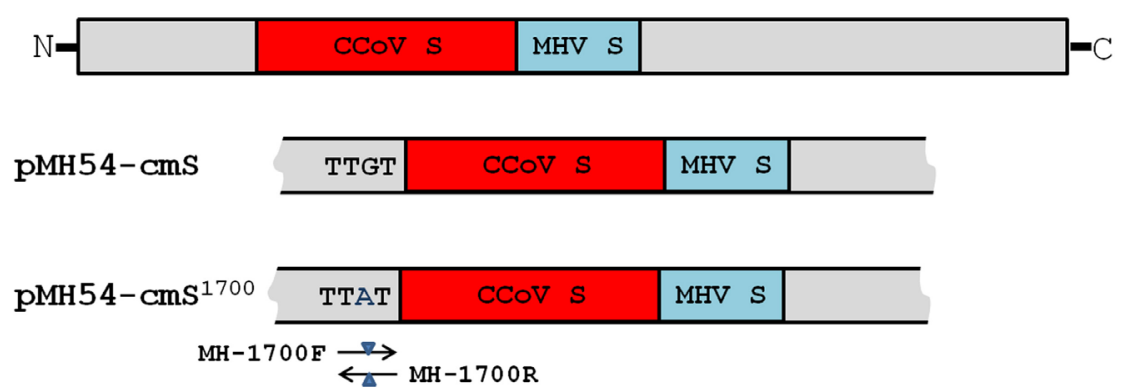

Figure 1. Schematic diagram of the primer design for site-directed mutagenesis. A. $t \mathrm{~N}-501 \mathrm{~F}, \mathrm{tN}-501 \mathrm{R}, \mathrm{tN}-959 \mathrm{~F}$, and $\mathrm{tN}-959 \mathrm{R}$ were for pIR-tN-EGFP. B. MH-1700F and MH-1700R were designed for $\mathrm{pMH} 54-\mathrm{cmS}^{1700}$. 


\section{PCR amplification of target-mutated fragment}

The PCR mixture $(50 \mu \mathrm{L})$ contained $1 \mathrm{ng}$ template, $0.5 \mu \mathrm{M}$ primer pair, $200 \mu \mathrm{M}$ dNTPs, $10 \mu \mathrm{L} 5 \mathrm{X}$ Q5 reaction buffer, and 1 U Q ${ }^{\mathrm{TM}}$ High-fidelity DNA polymerase (New England Biolabs). The reaction started with template pre-denaturation at $98^{\circ} \mathrm{C}$ for $30 \mathrm{~s}$, followed by 25 cycles at $98^{\circ} \mathrm{C}$ for $10 \mathrm{~s}, 55^{\circ} \mathrm{C}$ for $1 \mathrm{~s}$, and $72^{\circ} \mathrm{C}$ for 2 or 4 min according to the length of the template constructs (about $20-30 \mathrm{~s} / \mathrm{kb}$ ), and then an extension step at $72^{\circ} \mathrm{C}$ for $5 \mathrm{~min}$. PCR amplifications were confirmed by $1 \%$ agarose gel electrophoresis, and specific bands were observed under ultraviolet light. The reaction solution was stored at $4^{\circ} \mathrm{C}$. Using these same steps, we amplified a mutated fragment using Taq DNA polymerase (New England Biolabs).

\section{Digestion of template and transformation of digestion products}

PCR products were treated with the restriction enzyme DpnI (New England Biolabs) at $37^{\circ} \mathrm{C}$ for $2 \mathrm{~h}$ to digest the plasmid template. Next, $10 \mu \mathrm{L}$ digestion products were transformed into DH5 $\alpha$ E. coli cells by heat shock. The transformed mix was spread and inoculated onto Luria-Bertani plates containing kanamycin or ampicillin and then incubated at $37^{\circ} \mathrm{C}$ overnight. The number of colonies was counted to determine the number of colony forming units (CFU). Six colonies from each plate were grown in Luria-Bertani overnight and the plasmid DNA was extracted using Qiagen miniprep kits.

\section{Verification of plasmids}

The length of the plasmid was identified by restriction enzyme digestion. The introduction of site-directed mutagenesis was evaluated by plasmid DNA sequencing (Invitrogen, Carlsbad, CA, USA).

\section{RESULTS}

\section{Detection of gene mutation by PCR}

A large plasmid pMH54-cmS (12 kb) and common plasmid pIR-tN-EGFP $(6 \mathrm{~kb})$ were used as template plasmids in this study. The full-length pMH54-cmS ${ }^{1700}$ fragment $(12.3 \mathrm{~kb})$ obtained by PCR is shown in Figure 2A (lane 3). Figure 2A (lanes 1 and 2) shows the PCR screening for point mutations (S167R and G320E) in pIR-tN-EGFP.

\section{Counting of transformed colonies}

The plate was coated with $1 \mathrm{~mL}$ bacteria, and colonies were counted after incubation overnight. The number of pMH54-cmS ${ }^{1700}$ colonies was $2.7 \mathrm{CFU} / \mathrm{mL}$, while the number of pIR-tN ${ }^{501}$-EGFP was $37.8 \mathrm{CFU} / \mathrm{mL}$ and $52.5 \mathrm{CFU} / \mathrm{mL}$ for pIR-tN ${ }^{959}$-EGFP (Figure 2B).

\section{Verification of plasmid mutants}

Six clones per plasmid mutant were extracted and showed the expected sizes (Figure 3A). Plasmids pIR-tN ${ }^{501}$-EGFP and pIR-tN ${ }^{959}$-EGFP were subjected to restriction enzyme di- 
gestion (AvrII), generating 3 fragments $(3.1,2.4,1.0 \mathrm{~kb})$ (Figure 3B). Plasmid pMH54-cmS ${ }^{1700}$ was digested by EcoRI and PstI, generating 4 fragments (1.4, 2.7, 3.4, $4.7 \mathrm{~kb})$ (Figure 3B).

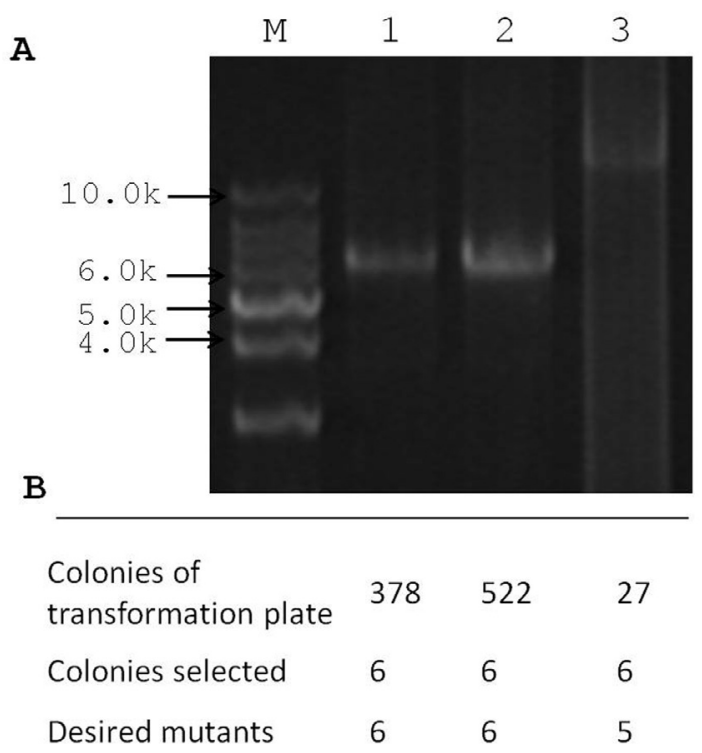

Figure 2. PCR amplification for single-site mutagenesis. A. Agarose gel electrophoresis of the PCRs indicating the amplification efficiency. Lane 1 was for pIR-tN501-EGFP, lane 2 was for pIR-tN959-EGFP, and lane 3 was for pMH54-cmS1700. Lane $M=$ DNA marker. B. Transformation and mutation efficiency of each reaction for pIRtN501-EGFP, pIR-tN959-EGFP, and pMH54-cmS1700.
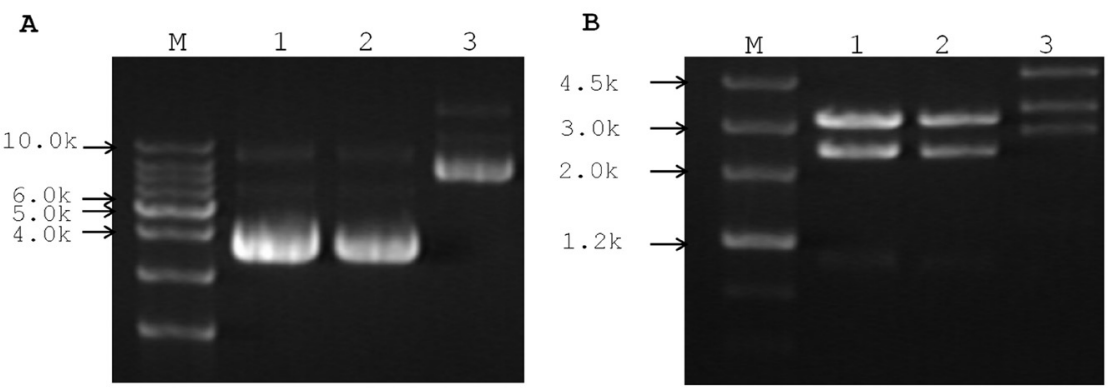

C

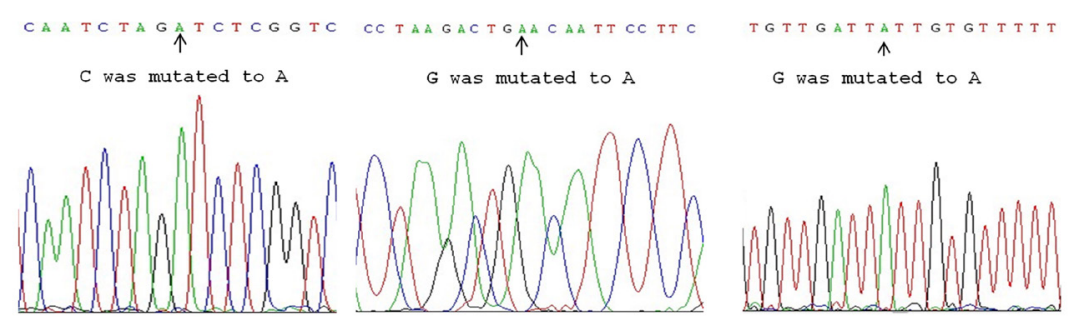

Figure 3. Verification of plasmid mutants. A. Agarose gel electrophoresis of three plasmid mutants. B. Restriction digestion of plasmids. Lane $1=$ pIR-tN ${ }^{501}$-EGFP was digested by AvrII; lane $2=$ pIR-tN ${ }^{959}$-EGFP was digested by AvrII; lane $3=\mathrm{pMH} 54-\mathrm{cmS}^{1700}$ was digested by EcoRI and PstI. C. Sequencing results of site-directed saturation mutagenesis. 
A total of 18 colonies were analyzed by sequencing (Figure 3C), only 1 of which was a sequencing error compared to the theoretical sequence. The success rate of site-directed mutagenesis using this protocol was $94.4 \%$, while the success rate of site-directed mutagenesis for the normal size plasmid $(6.4 \mathrm{~kb})$ was $100 \%$ and that of the large plasmid $(12.3 \mathrm{~kb})$ was $83.3 \%$.

\section{DISCUSSION}

\section{Amplification efficiency of Q5 ${ }^{\mathrm{TM}}$ High-fidelity DNA polymerase}

In this experiment, we found that Q5 ${ }^{\mathrm{TM}}$ DNA polymerase was efficient in the PCR amplification. The Taq DNA polymerase from New England Biolabs used for PCR only amplified a small fragment of $2 \mathrm{~kb}$, but was ineffective for long plasmids such as pMH54-cmS and pIR-tN-EGFP (data not shown). We found that the mutation products could be detected by agarose gel electrophoresis, while the QuikChange ${ }^{\mathrm{TM}}$ Site-Directed Mutagenesis System recommended continuing to the next step regardless of whether a visible fragment was detected by agarose gel electrophoresis. A visible fragment can increase the number of positive clones and increase the successes and failures of the procedure. In addition, other fidelity DNA polymerases produced by different companies have been reported to substitute and compared with QuikChange ${ }^{\mathrm{TM}}$, but whether $12 \mathrm{~kb}$ can be amplified is unknown (Picard et al., 1994; Liang et al., 1995; Adereth et al., 2005; Wu et al., 2013).

\section{Accuracy and mutation rate of $\mathrm{Q5}^{\mathrm{TM}}$ High-fidelity DNA polymerase}

Q5 ${ }^{\text {TM }}$ High-fidelity DNA polymerase possesses $3^{\prime} \rightarrow 5^{\prime}$ exonuclease activity, which can remove mismatched nucleotides during PCR. It is also fused to a processivity-enhancing Sso7d domain to support robust DNA amplification. Q5 ${ }^{\mathrm{TM}}$ DNA polymerase is supplied with an optimized buffer system to allow for robust amplification regardless of GC content. The 5X Q5 Reaction Buffer contains $2 \mathrm{mM} \mathrm{MgCl}{ }_{2}$ at final (1X) reaction concentration and is recommended for most routine applications. For GC-rich targets ( $\geq 65 \% \mathrm{GC})$, amplification can be improved by adding 5X Q5 High GC Enhancer. Q5 ${ }^{\mathrm{TM}}$ High-fidelity DNA polymerase is unlike typical, lower fidelity PCR enzymes. Q5 ${ }^{\mathrm{TM}}$ High-fidelity DNA polymerase has high fidelity with an error rate that is 100-fold lower than that of Taq DNA polymerase and 12-fold lower than that of Pyrococcus furiosus (PFU) DNA polymerase (NEB Information).

Our results demonstrate that the success rate of site-directed mutagenesis for the plasmid of typical size $(6.4 \mathrm{~kb})$ was 100 , while this value was $83.3 \%$ for a large plasmid $(12.3 \mathrm{~kb})$, with high fidelity and amplification efficiency by Q5 ${ }^{\mathrm{TM}}$ DNA polymerase to introduce mutations. We ensured the high efficiency by increasing the template concentration, reducing the number of PCR cycles, using a unique mutant primer design.

$D p n$ I is used to digest the plasmid template, removing the non-mutated background. $D p n I$ treatment can be used to obtain the correct mutant plasmid. Nearly all E. coli contain a gene encoding a 6-purine methylase, which methylates the 6-purine GATC sequence. DpnI can recognize methylated GATC and cut both methylated and hemi-methylated DNA (Shenoy and Visweswariah, 2003; Li et al., 2008). DNA synthesized in vitro is unmethylated, and thus can be selectively digested, while the newly synthesized PCR products are unaffected. To ensure complete template digestion, the digestion time could be extended to 2-3 $\mathrm{h}$ or more or the amount of $D p n \mathrm{I}$ can be increased (10-20 U). The same procedure without PCR primer 
can be conducted to evaluate whether $D p n$ I treatment was effective. If the plasmid template is completely digested by DpnI, the control plate that did not include primer in the PCR should show colonies.

\section{Simplicity and rapidity of the site-directed mutagenesis technique}

Our new protocol was successfully used to amply mutation fragments of different lengths in one step and is simple and easy to perform (Figure 4). The completely complementary mutagenic primer only annealed to and extended the original template in the cycle, such that $\mathrm{Q}^{\mathrm{TM}}$ DNA polymerase showed a linear amplification and not exponential amplification observed in typical PCR. This method ensures the greatest accuracy of template replication during temperature cycling. The residual template was digested by $D p n \mathrm{I}$, while the sub-chain with mutation forms double-stranded DNA containing a nick. The products were transformed into $E$. coli cells where the nick was ligated by host repair enzymes, and DH5 $\alpha$ E. coli cells were the most effective for the large plasmid. The entire process can be completed in only 1 day.

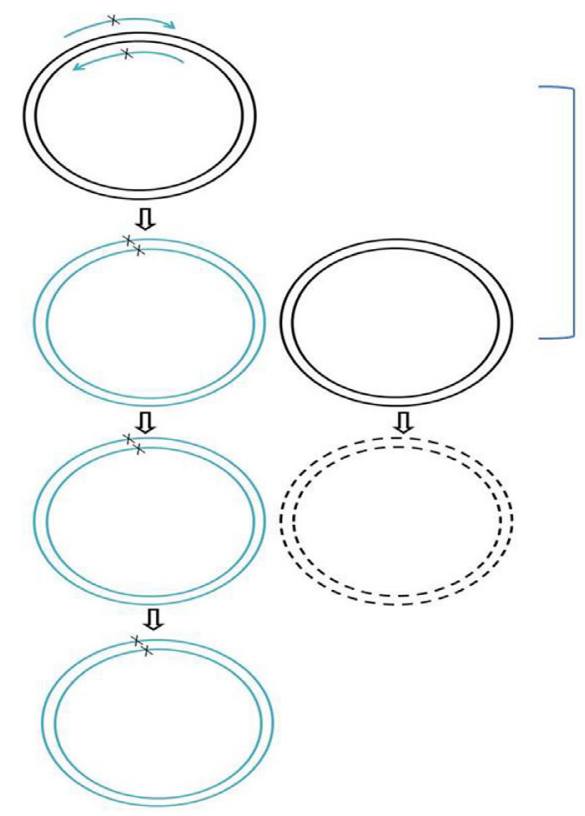

1. Synthesis of mutation chains by PCR

-Template DNA denaturation.

-Annealing between template DNA with the mutant primer.

-Primer extension under Q5 High-fidelity

DNA polymerase.

\section{DNA template digestion by $D p n I$}

-DpnI can identify methylated template plasmid

\section{Transformation}

- The the products are transformed into E. coli cells where the nick is ligated by host repair enzymes.

Figure 4. Schematic presentations using the one-step method.

We developed an economic method requiring only a single PCR amplification step. The Q $5^{\mathrm{TM}}$ DNA polymerase and $\mathrm{DpnI}$ must be purchased but are inexpensive, and other reagents include common laboratory reagents. The method is simple and convenient; the primer design requirements are simple, the process is easy to operate, and the entire process can be completed in only 1 day. The protocol is widely applicable for laboratory gene mutagenesis transformation, and is suitable for plasmids with a wide range of sizes, including 6-12 kb. 


\section{ACKNOWLEDGMENTS}

Research supported by open funds of the State Key Laboratory of Veterinary Etiological Biology, Lanzhou Veterinary Research Institute, Chinese Academy of Agricultural Sciences (\#SKLVEB2011KFKT003).

\section{REFERENCES}

Adereth Y, Champion KJ, Hsu T and Dammai V (2005). Site-directed mutagenesis using Pfu DNA polymerase and T4 DNA ligase. Biotechniques 38: 864-868.

Gillam S, Waterman K and Smith M (1975). Enzymatic synthesis of oligonucleotides of defined sequence. Addition of short blocks of nucleotide residues to oligonucleotide primers. Nucleic Acids Res. 2: 613-624.

Li J, Li C, Xiao W, Yuan D, et al. (2008). Site-directed mutagenesis by combination of homologous recombination and DpnI digestion of the plasmid template in Escherichia coli. Anal. Biochem. 373: 389-391.

Liang Q, Chen L and Fulco AJ (1995). An efficient and optimized PCR method with high fidelity for site-directed mutagenesis. PCR Methods Appl. 4: 269-274.

Picard V, Ersdal-Badju E, Lu A and Bock SC (1994). A rapid and efficient one-tube PCR-based mutagenesis technique using Pfu DNA polymerase. Nucleic Acids Res. 22: 2587-2591.

Qi D and Scholthof KB (2008). A one-step PCR-based method for rapid and efficient site-directed fragment deletion, insertion, and substitution mutagenesis. J. Virol. Methods 149: 85-90.

Shenoy AR and Visweswariah SS (2003). Site-directed mutagenesis using a single mutagenic oligonucleotide and $D p n$ I digestion of template DNA. Anal. Biochem. 319: 335-336.

Shinde D, Lai Y, Sun F and Arnheim N (2003). Taq DNA polymerase slippage mutation rates measured by PCR and quasi-likelihood analysis: (CA/GT)n and (A/T)n microsatellites. Nucleic Acids Res. 31: 974-980.

Wu D, Guo X, Lu J, Sun X, et al. (2013). A rapid and efficient one-step site-directed deletion, insertion, and substitution mutagenesis protocol. Anal. Biochem. 434: 254-258.

Zoller MJ and Smith M (1987). Oligonucleotide-directed mutagenesis: a simple method using two oligonucleotide primers and a single-stranded DNA template. Methods Enzymol. 154: 329-350. 